\title{
Sur une propriété des fonctions de M. Hamel.
}

\section{Par}

\author{
W. Sierpiński (Varsovie).
}

Le but de cette Note est démontrer lo théorème suivant qui m'a été suggeré par une question. posée par mon élève, M. O. N i k od y m:

Theorème: Une fonction discontinue d'une variable rélle $f(x)$ satisfaisant d̀ l'équation fonctionnelle

$$
f(x+y)=f(x)+f(y)
$$

ne peut être majorée par aucune fonction mesurable.

De ce théorème résulte tout de suite que si $f(x)$ est une solution de l'équation (1) et s'il existe une fonction mesurable $(L), \varphi(x)$, telle que $f(x) \leqslant \varphi(x)$ (pour tout $x$ réel), $f(x)$ est une fonstion continue (donc, d'après $\mathrm{Oau}$ chy, de la forme $A x$ ). En particulier, tonte fonction mesurable $(L)$, satisfaisant a l'équation (1), est continue").

Soient: $f(x)$ une fonction discontinue, satisfaisant à l'équation (1) $\left.{ }^{4}\right)$, $(a, b)$ - un intervalle donné, $A$ - un nombre réel donné quelconque, $\varepsilon$ un nombre positif. Nous prouverons que la mesure extérieure (lebesguienne) de l'ensemble

$$
E(A, a, b)=\mathrm{E}[f(x)>A, a \leqslant x \leqslant b]
$$

est

$$
m_{0} E(A, a, b)=b-a
$$

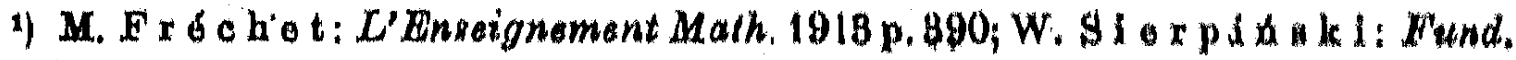
Math t. 1, p 116; 8. Banach: Fund. Math. t, 1, p. 183; H. Hi a n Theoris der resllen fiunktionan I (Berlin 1981), p. 588.

1) L'existence des molutions discontinuel do l'équation (1) an for dómontrón on 1906 par M. H a mol (a l'uide da theorémo de M. Z on mo l. o): Math. Ann. 60, p. 460. 
Nons avons évidemment

done

$$
\mathrm{E}[a \leqslant x \leqslant b]=\sum_{n=1}^{\infty} E(-n, a, b),
$$

$$
m_{\mathrm{a}} \mathrm{E}[a \leqslant x \leqslant b]=\lim _{N=\infty} m_{c} \sum_{n=1}^{N} E(-n, a, b),
$$

c'est-à-dire

$$
b-a=\lim _{n \rightarrow \infty} m_{e} E(-N, a, b):
$$

il existe donc un indice $N$ tel que

$$
m_{a} E(-N, a, b)>b-a-\varepsilon .
$$

$f(x)$ étant une fonction, satisfaisant à l'équation (1), il existe, comme on sait, un nombre $h$, tel que

$$
\begin{gathered}
0<h<\varepsilon, h<b-a \\
\left.f(h)>N+A^{1}\right) .
\end{gathered}
$$$$
\text { et }
$$

D'après (4) et (5) nous trouvons sans peine:

$$
m_{e} E(-N, a, b-h)>b-a-2 \varepsilon .
$$

Désignons par $H$ l'ensemble qu'ọ obtient par une translation de l'ensemble $E(-N, a, b-h)$ de longueur $h$ : l'ensemble $H$ sera évidemment contenu dans l'intervalle $(a, b)$, et nous aurons, d'après (7):

$$
m_{e} H>b-a-2 \varepsilon \text {. }
$$

Soit $x$ un point de l'ensemble $H$ : le point $x-h$ appartient done à $E(-N, a, b-h)$, donc

$$
f(x-h)>-N \text {. }
$$

Or, d'après $(1), f(x)=f(x-h)+f(h)$, donc, d'après (9) et (6):

$$
f(x)>A
$$

ce qui prouve que $x$ (qui est un point de l'intervalle $(a, b$ ), comme point de $H)$ appartient à l'ensemble $E(A, a, b)$.

1) V. p. e. G D a r b o a : Math. Ann. 17, p. 56 . 
Nous avons donc démontré que

$$
H \subset E(A, a, b) \text {; }
$$

l'inégalité (8) donne donc:

$$
m_{e} E(A, a, b)>b-a-2 \varepsilon
$$

le nombre positif $\varepsilon$ étant arbitraire, cette inégalité donne:

$$
m_{i} E(A, a, b) \geqslant b-a
$$

ce qui prouve la formule (3).

Admettons maintenant qu'il existe une fonction mesurable (finie) $\varphi(x)$, telle que

$$
f(x) \leqslant \varphi(x)
$$

pour tout $x$ réel.

On aurait donc, pour tout $A$ réel et tout intervalle $(a, b)$ :

$$
\mathrm{E}[f(x)>A, a \leqslant x \leqslant b] \subset \mathrm{E}[\varphi(x)>A, a \leqslant x \leqslant b],
$$

donc, d'après (3):

$$
\mathrm{E} \mid \varphi(x)>A, a \leqslant x \leqslant b]=b-a,
$$

pour tout $A$ réel ce qui est impossible, puisque $\varphi(x)$ est une fonction mesurable.

Notre théorème est ainsi démontré.

Remarquons encore qu'on pourrait démontrer sans peine le théorème suivant: Toute fonction $f(x)$ satisfaisant à l'équation fonctionnelle (1) et à la condition de Baire ${ }^{1}$ est continue.

(En effet on démontre sans peine que si $f(x)$ est une solution discontinue de l'équation (1), les ensembles $\mathrm{E} \mid f(x)>0\}$ et $\mathrm{E} \mid f(x)<0]$ sont de deuxième catégorie dans tout inlervalle, d'où résulte que $f(x)$ ne satisfait à la condition de Baire dans aucun intervalle).

1) C. à d ponctuellement discontinue sur tout ensemble parfait quand on néglige los ensembles de $1^{\text {re }}$ catégorie par rapport à cet ensemble parfait. 